\title{
Oral health of children in association with gender and mothers' education: A comparative cross-sectional study
}

\author{
Heba Ashi
}

Department of Dental Public Health, Faculty of Dentistry, King Abdulaziz University, Jeddah, Saudi Arabia

Correspondence to: Heba Ashi (E-mail: hmashi@kau.edu.sa)

(Submitted: 12 December 2020 - Revised version received: 28 December 2020 - Accepted: 19 January 2020 - Published online: 26 February 2021)

\begin{abstract}
Objective To evaluate the association between gender and mothers' education, and the oral hygiene of their children.

Methods This comparative cross-sectional study included 531 children in Jeddah, Saudi Arabia. Arabic was chosen for this survey to avoid possibilities of language barriers, followed by data analysis and segregation from the survey. Students were stratified according to gender and their mother's education. Data were analyzed using the Statistical Package for the Social Sciences (SPSS), version 25.

Results Brushing twice daily, using the dental floss, using fluoridated toothpaste, using mouthwash regularly, and bruxism were higher among females than males ( $58 \%$ vs. $28 \%, 28.2 \%$ vs. $10.9 \%, 71 \%$ vs. $30 \%, 55 \%$ vs. $35 \%$ and $16.5 \%$ vs. $9.8 \%$, respectively) with a significant difference $(p<0.001,<0.001,<0.001,<0.001$ and $<0.001$, respectively). Meanwhile, using toothpick, clenching, and biting on hard objects were higher among males than females ( $49.3 \%$ vs. $34.9 \%, 21.7 \%$ vs. $7.1 \%$ and $46.7 \%$ vs. $34.9 \%$, respectively) with a significant difference $(<0.001$ and $<0.001$, respectively). Going to dentists during the last 3 months was significantly higher among females than males $(47 \%$ vs. $33 \%)$, with a significant difference $(\mathrm{p}<0.001)$. On the other hand, going to dentists every 6 months was notably higher among males than females $(22 \%$ vs.. $17 \%)$ ( $p<0.001)$. Regular check-ups were higher among females ( $27.8 \%$ vs. $16.7 \%)$, with a significant difference ( $p=0.007)$. In accordance with the mothers' education, brushing more than twice daily, manual toothbrush, electrical toothbrush, dental floss, and using toothpaste were significantly higher among those who received a university and post-graduate education $(p<0.001,<0.001,<0.001$, and 0.049 , respectively). Going to dentists during the previous 3 and 6 months was higher among those who received a university and post-graduate education $(p=0.001)$.

Conclusions Girls and children whose mothers had a university and post-graduate education had better attitudes toward oral hygiene. Therefore, we highly recommend more oral hygiene health education programs, especially for mothers with less education.

Keywords Oral health, Oral hygiene, Mothers education
\end{abstract}

\section{Introduction}

The World Health Organization (WHO) states that oral health is considered a part of the general well-being and is an essential element for good quality of life. ${ }^{1}$ Good oral health helps us speak, smile, taste, chew, swallow, and express ourselves confidently. ${ }^{2,3}$ Besides, it affects appearance, by allowing people to perform their daily activities without psychological or social limitations (Shah and ElHaddad, 2015)not many people are aware of the relationship of smoking with potential oral diseases. Therefore, the aims of this study were to analyze oral hygiene behavior, smoking habits, and perceived oral health problems among a sample of university students in Al-Kharj, Saudi Arabia. Materials and Methods: A self-administered questionnaire about oral hygiene behavior, smoking, and perceived oral health problems was developed. The questionnaires were mainly distributed in Medical, Dental, and Pharmacy colleges of the university. Questionnaires completed at other colleges were included under the term "other colleges." Results: Overall, 380 questionnaires were returned. Majority of the students $(92.4 \%$. This depends on several factors, such as personal attitudes, behaviors, and knowledge. In the literature, the focus was mainly on the cure for oral diseases rather than prevention. ${ }^{4}$

Several behavioral models, such as the health belief model and reasoned action theory, established the significant role of knowledge in behavioral changes explanation. ${ }^{5}$ Parents' knowledge and attitudes play an essential role in raising the awareness in their children and encouraging them to have a healthy life. ${ }^{6}$ Additionally, parents' involvement in health promotion programs can improve children's health, and further, the mothers' higher education elevated their children's oral health. ${ }^{7}$ Moreover, the lack of the mothers' knowledge about oral hygiene may be one of the contributing factors for the dental caries' high prevalence among children. ${ }^{8}$ Therefore, poor parenting may be one of the etiological factors for dental caries. ${ }^{9}$ It was reported that children of mothers with positive oral attitudes were more likely to brush their teeth twice daily (Z, Virtanen, Ghofranipour, \& Murtomaa, 2008).

So far, no studies have been conducted in Saudi Arabia to assess the effect of mothers' education on the oral hygiene of children. The study aimed to evaluate the association between gender and the mothers' education, and the oral hygiene of their children.

\section{Methods}

This comparative cross-sectional study included 531 children in Jeddah, Saudi Arabia. An electronic database search was performed using Google Scholar and PubMed to gather background information and data related to the research question and to determine the knowledge gap. Arabic was chosen for this survey to avoid possibilities of language barriers, followed by data analysis and segregation from the survey. Students were stratified according to their gender and their mother's education. 
Data were analyzed using the Statistical Package for the Social Sciences (SPSS), version 25. Categorical variables are presented as numbers and percentages. Results were compared using the Chi-square test and the Monto Carlo test. All the tests were 2 -tailed, and a p-value $<0.05$ was considered statistically significant.

Ethical considerations: The study was approved by the Research Ethics Committee of the Faculty of Dentistry (REC-FD), and consent was approved by the parents before any data collection.

\section{Results}

Out of the 531 students, the mothers of 250 (47.1\%) students had a university and post-graduate education, whereas the number of students with fathers having a university or post-graduate education were 353 (66.5\%) (Table 1).

According to oral hygiene habits, when comparing both genders, brushing twice daily, using the dental floss, fluoridated toothpaste, and mouthwash regularly, and bruxism were higher among females than males (58\% vs. $28 \%, 28.2 \%$ vs. $10.9 \%, 71 \%$ vs. $30 \%, 55 \%$ vs. $35 \%$ and $16.5 \%$ vs. $9.8 \%$, respectively) with a significant difference $(\mathrm{p}<.001,<.001,<.001$, $<.001$, and <.001, respectively). However, using toothpick, clenching, and biting on hard objects were higher among males than females $(49.3 \%$ vs. $34.9 \%, 21.7 \%$ vs. $7.1 \%$, and $46.7 \%$ vs. $34.9 \%$ respectively) with a significant difference $(<.001$ and $<.001$, respectively) (Table 2, Figs 1, 2, 3, 4, and 5).

When comparing dental history between both sexes, going to dentists during the last 3 months was higher among females than males (47\% vs. $33 \%$ ), with a significant difference $(\mathrm{p}<.001)$. Contrarily, going to dentists every 6 months was significantly higher among males than females $(22 \%$ vs. 17\%) $(\mathrm{p}<.001)$. Additionally, regular check-ups were higher among females (27.8\% vs. $16.7 \%)$, with a significant difference $(\mathrm{p}=.007)$ (Table 3, Figs 6 and 7).

While evaluating the oral hygiene habits with regard to the mothers' education, brushing more than twice daily, using a manual/electrical toothbrush, using dental floss, and using toothpaste were significantly higher among those with a university and post-graduate education $(\mathrm{p}<.001,<.001,<.001$, and $<.01$, respectively) (Table 4, Figs 8 and 9 ).

When comparing dental history according to the mothers' education, going to the dentist during the previous 3 and 6 months was higher among those with a university and post-graduate education ( $\mathrm{p}=.001)$ (Table 5$)$.

\section{Table 1. Family education of the sample $(\mathbf{n}=\mathbf{5 3 1})$.}

\begin{tabular}{|c|c|c|c|}
\hline & & $\mathbf{N}$ & Percent \\
\hline \multirow{3}{*}{$\begin{array}{l}\text { 1. What } \\
\text { education does } \\
\text { your mother } \\
\text { have? }\end{array}$} & University and postgraduate & 250 & 47.1 \\
\hline & Secondary and preparatory & 75 & 14.1 \\
\hline & Others & 206 & 38.8 \\
\hline \multirow{3}{*}{$\begin{array}{l}\text { 2. What } \\
\text { education does } \\
\text { your father } \\
\text { have? }\end{array}$} & University and postgraduate & 353 & 66.5 \\
\hline & Secondary and preparatory & 31 & 5.8 \\
\hline & Others & 147 & 27.7 \\
\hline
\end{tabular}

Note. All variables are summarized as percentage

\section{Discussion}

This comparative cross-sectional study was conducted at King Abdul-Aziz University and included 531 children. It assessed the association between mothers' education and gender and oral health practices among their children.

The outline suggests that females and children of mothers with a university and post-graduate education showed better attitudes toward oral hygiene habits and dentist visits.

In the gender-based oral health comparison in our sample, brushing twice daily, using the dental floss, fluoridated toothpaste, and mouthwash regularly, and bruxism was higher among females than males. However, using toothpick, clenching, biting on hard objects was higher among males than females. Therefore, we can conclude that females are more committed to oral health and hygiene. This could be due to their aesthetic concerns and the presentation of their smile. This finding is similar to a study by Singh et $\mathrm{al}^{10}$, who found that the male gender was significantly related to dental caries. Additionally, a study by Kumar et $\mathrm{al}^{11}$ found that females had better knowledge and oral health practices than males. ${ }^{11}$ Similarly, a study by Jaber et al found that males had good knowledge but poor practice toward oral health. ${ }^{12}$

When comparing the dental history and commitment to visits between both genders, females showed more commitment than males, which is similar to a study by McDonald, ${ }^{13}$ who also reported similar results.

Regarding the oral hygiene habits, in association with the mothers' education, brushing twice daily, using the manual toothbrush and electrical toothbrush, was higher among those with a university and post-graduate education. This is on par with a study by Hallas et $\mathrm{al}^{8}$, who found that lack of knowledge of mothers regarding oral hygiene might be one of the contributing factors for the high dental caries prevalence in children. Additionally, a study by Z, Virtanen, Ghofranipour, and Murtomaa (2008) found that children of mothers with positive attitudes were more likely to brush their teeth twice daily.

This study showed the difference in oral health and attitudes among girls and boys, children of mothers with a university or post-graduate education, and with secondary or primary education. Thus, we highly recommend more oral hygiene health education programs, especially for mothers with less education.

Data were collected via an online survey that had some restrictions, such as the likelihood of inaccurate information, as the survey is in the form of multiple-choice questions. Respondents may not feel comfortable providing answers that present themselves in an unfavorable manner.

\section{Conclusion}

Females had a better attitude toward oral hygiene than males. Moreover, children whose mothers had a university and post-graduate education also had better attitudes toward oral hygiene habits and dentist visits. Thus, we highly recommend more oral hygiene health education programs, especially for mothers with less education. The literature review suggests that no studies have been conducted in Saudi Arabia to assess the effects of the mothers' education on oral hygiene of children. Further research is needed to determine oral health problems based on clinical examination and comprehensive 


\begin{tabular}{|c|c|c|c|c|c|c|}
\hline & & \multicolumn{2}{|c|}{ boys } & \multicolumn{2}{|c|}{ girls } & \multirow{2}{*}{ P-value } \\
\hline & & $\mathbf{N}$ & $\%$ & $\mathbf{N}$ & $\%$ & \\
\hline \multirow{6}{*}{$\begin{array}{l}\text { 1. How often do you } \\
\text { brush your teeth? }\end{array}$} & Never & 2 & $1 \%$ & 0 & $0 \%$ & \multirow[t]{6}{*}{$<0.001^{*}$} \\
\hline & Once or a few times a week & 43 & $16 \%$ & 4 & $2 \%$ & \\
\hline & Once a day & 119 & $43 \%$ & 30 & $12 \%$ & \\
\hline & Twice a day & 78 & $28 \%$ & 149 & $58 \%$ & \\
\hline & More than twice a day & 23 & $8 \%$ & 61 & $24 \%$ & \\
\hline & Other & 11 & $4 \%$ & 11 & $4 \%$ & \\
\hline \multirow{7}{*}{$\begin{array}{l}\text { 2. What do you use to } \\
\text { brush your teeth? }\end{array}$} & Manual toothbrush & 233 & $84.4 \%$ & 217 & $85.1 \%$ & \multirow[t]{7}{*}{$0.003^{*}$} \\
\hline & Manual and electrical toothbrush & 6 & $2.2 \%$ & 2 & $0.8 \%$ & \\
\hline & Manual toothbrush and miswak & 12 & $4.3 \%$ & 2 & $0.8 \%$ & \\
\hline & Electrical toothbrush & 20 & $7.2 \%$ & 26 & $10.2 \%$ & \\
\hline & Miswak & 0 & $0.0 \%$ & 5 & $2.0 \%$ & \\
\hline & None & 2 & $0.7 \%$ & 3 & $1.2 \%$ & \\
\hline & Other & 3 & $1.1 \%$ & 0 & $0.0 \%$ & \\
\hline \multirow{8}{*}{$\begin{array}{l}\text { 3. Which of the } \\
\text { following product } \\
\text { do you use to clean } \\
\text { between your teeth? }\end{array}$} & Dental floss & 30 & $10.9 \%$ & 72 & $28.2 \%$ & \multirow[t]{8}{*}{$<0.001 *$} \\
\hline & Dental floss and Interdental brush & 5 & $1.8 \%$ & 7 & $2.7 \%$ & \\
\hline & Dental floss and Toothpick & 5 & $1.8 \%$ & 2 & $0.8 \%$ & \\
\hline & Interdental brush & 26 & $9.4 \%$ & 21 & $8.2 \%$ & \\
\hline & Interdental brush and Toothpick & 6 & $2.2 \%$ & 0 & $0.0 \%$ & \\
\hline & Toothpick & 136 & $49.3 \%$ & 89 & $34.9 \%$ & \\
\hline & None & 65 & $23.6 \%$ & 60 & $23.5 \%$ & \\
\hline & Other & 3 & $1.1 \%$ & 4 & $1.6 \%$ & \\
\hline \multirow{2}{*}{$\begin{array}{l}\text { 4. Do you use any } \\
\text { toothpaste while } \\
\text { brushing? }\end{array}$} & Yes & 269 & $97 \%$ & 236 & $93 \%$ & \multirow[t]{2}{*}{$0.009 * *$} \\
\hline & No & 7 & $3 \%$ & 19 & $7 \%$ & \\
\hline \multirow{3}{*}{$\begin{array}{l}\text { 5. What kind of } \\
\text { toothpaste do you use? }\end{array}$} & Fluoridated & 82 & $30 \%$ & 182 & $71 \%$ & \multirow[t]{3}{*}{$<0.001 *$} \\
\hline & Non-fluoridated & 8 & $3 \%$ & 0 & $0 \%$ & \\
\hline & Do not know & 184 & $67 \%$ & 73 & $29 \%$ & \\
\hline \multirow{2}{*}{$\begin{array}{l}\text { 6.Do you use any } \\
\text { mouthwash regularly? }\end{array}$} & Yes & 96 & $35 \%$ & 141 & $55 \%$ & \multirow[t]{2}{*}{$<0.001^{* *}$} \\
\hline & No & 178 & $65 \%$ & 114 & $45 \%$ & \\
\hline \multirow{4}{*}{$\begin{array}{l}\text { 7. Do you have any } \\
\text { of the following oral } \\
\text { habits? }\end{array}$} & Bruxism & 27 & $9.8 \%$ & 42 & $16.5 \%$ & \multirow[t]{4}{*}{$<0.001^{* *}$} \\
\hline & Clenching & 60 & $21.7 \%$ & 18 & $7.1 \%$ & \\
\hline & Biting on a hard object & 129 & $46.7 \%$ & 89 & $34.9 \%$ & \\
\hline & Other & 60 & $21.7 \%$ & 106 & $41.6 \%$ & \\
\hline
\end{tabular}

Note. All variables are summarized as percentage.

The test of significance was carried out at 0.05 level.

*Monto Carlo test was used.

${ }^{* *}$ Chi-Square test was used.

Significant results are in bold. 


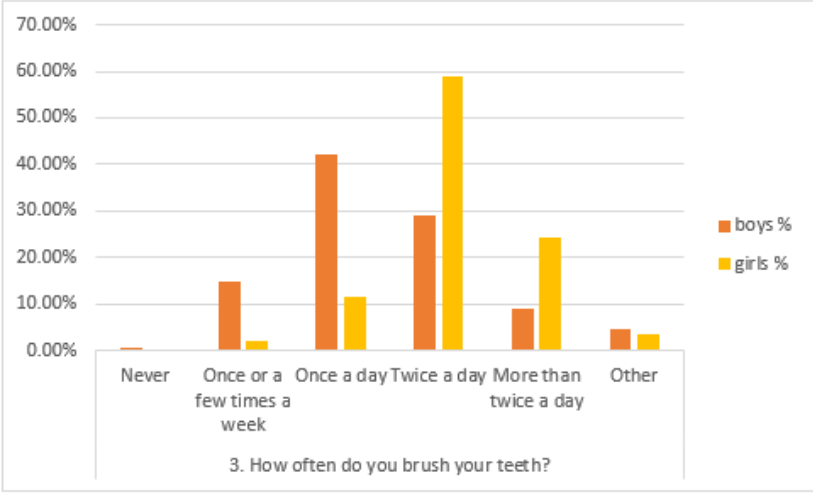

Fig. 1 Toothbrushing according to gender.2017 $(n=8665)$.

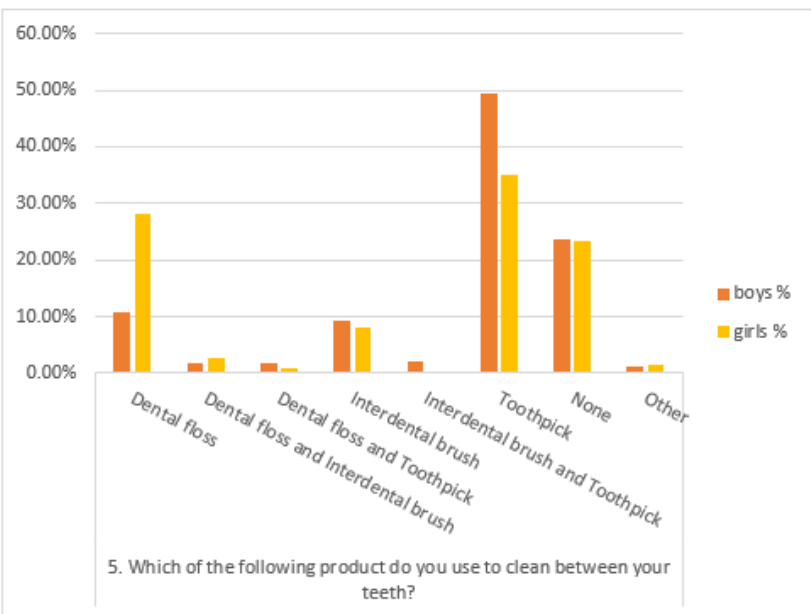

Fig. 2 Cleaning product use according to gender.



Fig. 3 Toothpaste type according to gender.

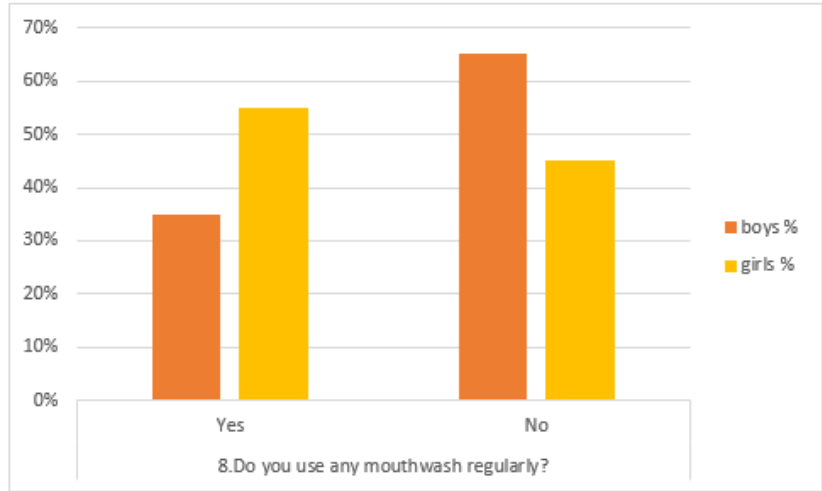

Fig. 4 Mouthwash use according to gender.

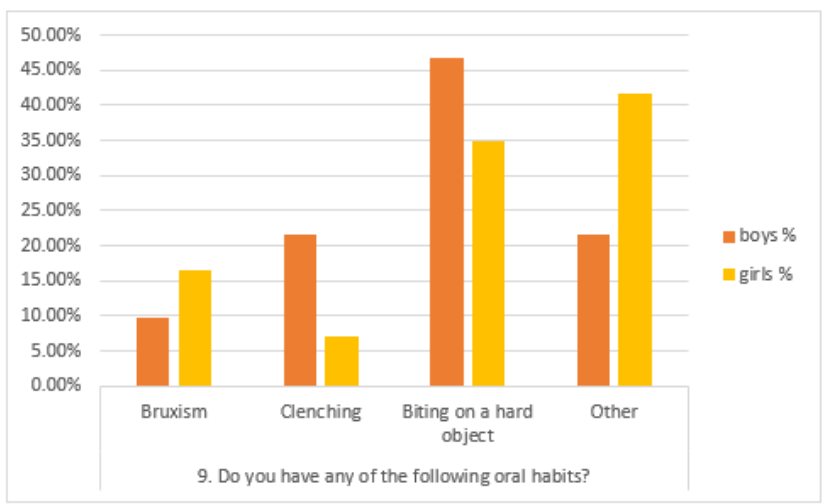

Fig. 5 Oral habits according to gender.

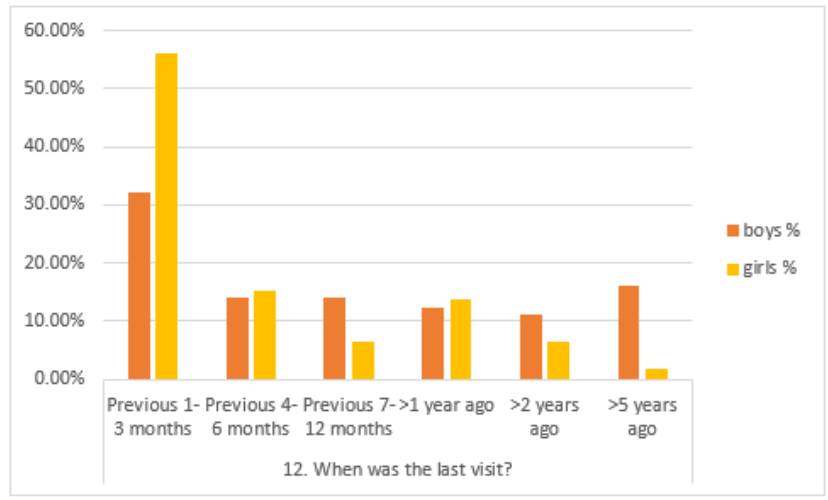

Fig. 6 Dentist last visit according to gender. 


\begin{tabular}{|c|c|c|c|c|c|c|}
\hline & & \multicolumn{2}{|c|}{ Boys } & \multicolumn{2}{|c|}{ Girls } & \multirow{2}{*}{ P-value } \\
\hline & & $\mathbf{N}$ & $\%$ & $\mathbf{N}$ & $\%$ & \\
\hline \multirow{2}{*}{$\begin{array}{l}\text { 1. Have you ever been } \\
\text { to a dentist? }\end{array}$} & Yes & 266 & $96 \%$ & 250 & $98 \%$ & \multirow[t]{2}{*}{$0.248^{* *}$} \\
\hline & No & 10 & $4 \%$ & 5 & $2 \%$ & \\
\hline \multirow{5}{*}{$\begin{array}{l}\text { 2. If yes, how often do } \\
\text { you visit a dentist? }\end{array}$} & Every 6 months & 61 & $22 \%$ & 43 & $17 \%$ & \multirow[t]{5}{*}{$0.001 *$} \\
\hline & Every year & 5 & $2 \%$ & 16 & $6 \%$ & \\
\hline & Irregularly & 61 & $22 \%$ & 39 & $15 \%$ & \\
\hline & Only when in pain & 119 & $43 \%$ & 109 & $43 \%$ & \\
\hline & Other & 30 & $11 \%$ & 45 & $18 \%$ & \\
\hline \multirow{6}{*}{$\begin{array}{l}\text { 3. When was the last } \\
\text { visit? }\end{array}$} & Previous $1-3$ months & 90 & $33 \%$ & 121 & $47 \%$ & \multirow[t]{6}{*}{$<0.001^{* *}$} \\
\hline & Previous 4-6 months & 39 & $14 \%$ & 44 & $17 \%$ & \\
\hline & Previous 7-12 months & 41 & $15 \%$ & 19 & $7 \%$ & \\
\hline & $>1$ year ago & 33 & $12 \%$ & 38 & $15 \%$ & \\
\hline & $>2$ years ago & 31 & $11 \%$ & 23 & $9 \%$ & \\
\hline & $>5$ years ago & 42 & $15 \%$ & 10 & $4 \%$ & \\
\hline \multirow{6}{*}{$\begin{array}{l}\text { 4. What was the } \\
\text { purpose of the visit? }\end{array}$} & Regular check-up (dental examination) & 46 & $16.7 \%$ & 71 & $27.8 \%$ & \multirow[t]{6}{*}{$0.007^{* *}$} \\
\hline & Cleaning the teeth & 40 & $14.5 \%$ & 35 & $13.7 \%$ & \\
\hline & Filling & 68 & $24.6 \%$ & 46 & $18.0 \%$ & \\
\hline & Extraction & 33 & $12.0 \%$ & 18 & $7.1 \%$ & \\
\hline & Orthodontic treatment & 63 & $22.8 \%$ & 51 & $20.0 \%$ & \\
\hline & Other & 26 & $9.4 \%$ & 34 & $13.3 \%$ & \\
\hline
\end{tabular}

Note. All variables are summarized as percentage.

The test of significance was carried out at 0.05 level.

* Monto Carlo test was used.

${ }^{* *}$ Chi- Square test was used.

Significant results are in bold.

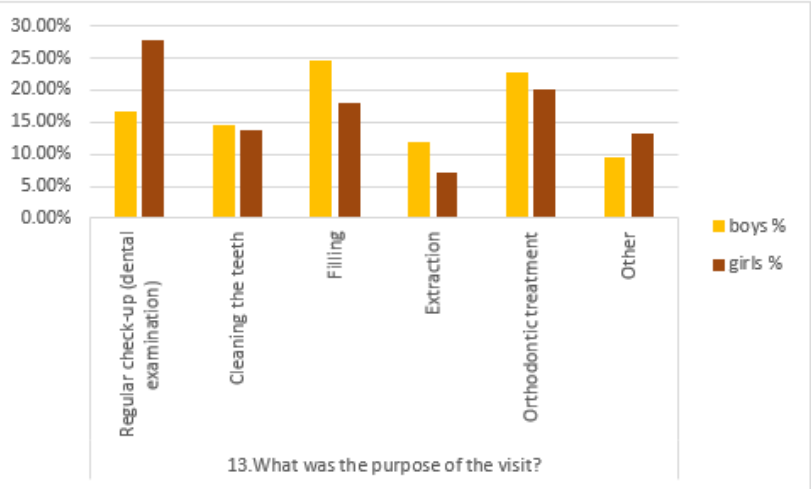

Fig. 7 Purpose of the visit according to gender.

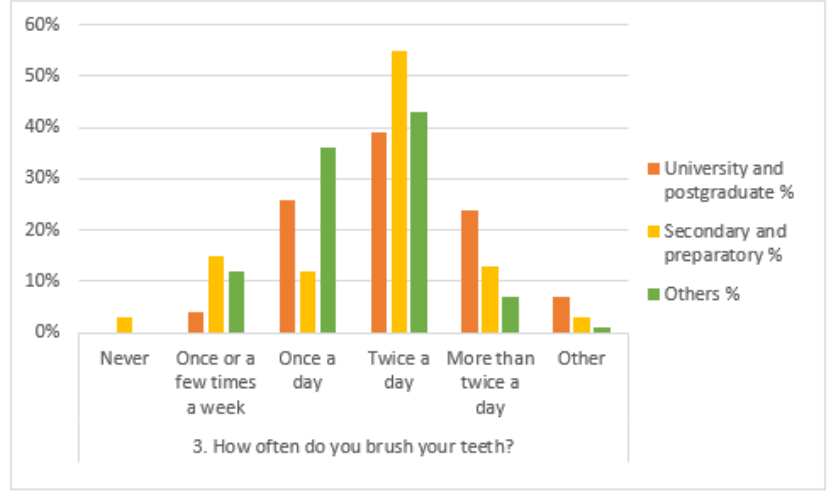

Fig. 8 Toothbrushing according to mothers' education. 


\begin{tabular}{|c|c|c|c|c|c|c|c|c|}
\hline & & \multicolumn{2}{|c|}{$\begin{array}{l}\text { University and } \\
\text { postgraduate }\end{array}$} & \multicolumn{2}{|c|}{$\begin{array}{c}\text { Secondary and } \\
\text { preparatory }\end{array}$} & \multicolumn{2}{|c|}{ Others } & \multirow[t]{2}{*}{ P-value } \\
\hline & & N & $\%$ & N & $\%$ & N & $\%$ & \\
\hline \multirow{6}{*}{$\begin{array}{l}\text { 1. How often do } \\
\text { you brush your } \\
\text { teeth? }\end{array}$} & Never & 0 & $0 \%$ & 2 & $3 \%$ & 0 & $0 \%$ & \multirow[t]{6}{*}{$<0.001^{*}$} \\
\hline & Once or a few times a week & 11 & $4 \%$ & 11 & $15 \%$ & 25 & $12 \%$ & \\
\hline & Once a day & 65 & $26 \%$ & 9 & $12 \%$ & 75 & $36 \%$ & \\
\hline & Twice a day & 97 & $39 \%$ & 41 & $55 \%$ & 89 & $43 \%$ & \\
\hline & More than twice a day & 60 & $24 \%$ & 10 & $13 \%$ & 14 & $7 \%$ & \\
\hline & Other & 17 & $7 \%$ & 2 & $3 \%$ & 3 & $1 \%$ & \\
\hline \multirow{7}{*}{$\begin{array}{l}\text { 2. What do you } \\
\text { use to brush your } \\
\text { teeth? }\end{array}$} & Manual toothbrush & 207 & $82.8 \%$ & 66 & $88.0 \%$ & 177 & $85.9 \%$ & \multirow[t]{7}{*}{$<0.001^{*}$} \\
\hline & Manual and electrical toothbrush & 0 & $0.0 \%$ & 0 & $0.0 \%$ & 8 & $3.9 \%$ & \\
\hline & Manual toothbrush and miswak & 2 & $0.8 \%$ & 0 & $0.0 \%$ & 12 & $5.8 \%$ & \\
\hline & Electrical toothbrush & 33 & $13.2 \%$ & 7 & $9.3 \%$ & 6 & $2.9 \%$ & \\
\hline & Miswak & 5 & $2.0 \%$ & 0 & $0.0 \%$ & 0 & $0.0 \%$ & \\
\hline & None & 0 & $0.0 \%$ & 2 & $2.7 \%$ & 3 & $1.5 \%$ & \\
\hline & Other & 3 & $1.2 \%$ & 0 & $0.0 \%$ & 0 & $0.0 \%$ & \\
\hline \multirow{8}{*}{$\begin{array}{l}\text { 3. Which of } \\
\text { the following } \\
\text { product do you } \\
\text { use to clean } \\
\text { between your } \\
\text { teeth? }\end{array}$} & Dental floss & 69 & $27.6 \%$ & 15 & $20.0 \%$ & 18 & $8.7 \%$ & \multirow[t]{8}{*}{$<0.001^{*}$} \\
\hline & Dental floss and Interdental brush & 9 & $3.6 \%$ & 0 & $0.0 \%$ & 3 & $1.5 \%$ & \\
\hline & Dental floss and Toothpick & 2 & $0.8 \%$ & 2 & $2.7 \%$ & 3 & $1.5 \%$ & \\
\hline & Interdental brush & 28 & $11.2 \%$ & 4 & $5.3 \%$ & 15 & $7.3 \%$ & \\
\hline & Interdental brush and Toothpick & 2 & $0.8 \%$ & 0 & $0.0 \%$ & 4 & $1.9 \%$ & \\
\hline & Toothpick & 89 & $35.6 \%$ & 38 & $50.7 \%$ & 98 & $47.6 \%$ & \\
\hline & None & 49 & $19.6 \%$ & 14 & $18.7 \%$ & 62 & $30.1 \%$ & \\
\hline & Other & 2 & $0.8 \%$ & 2 & $2.7 \%$ & 3 & $1.5 \%$ & \\
\hline \multirow{2}{*}{$\begin{array}{l}\text { 4. Do you use any } \\
\text { toothpaste while } \\
\text { brushing? }\end{array}$} & Yes & 236 & $94 \%$ & 68 & $91 \%$ & 201 & $98 \%$ & \multirow[t]{2}{*}{$0.049^{*}$} \\
\hline & No & 14 & $6 \%$ & 7 & $9 \%$ & 5 & $2 \%$ & \\
\hline \multirow{3}{*}{$\begin{array}{l}\text { 5. What kind of } \\
\text { toothpaste do } \\
\text { you use? }\end{array}$} & Fluoridated & 144 & $58 \%$ & 45 & $60 \%$ & 75 & $37 \%$ & \multirow[t]{3}{*}{$<0.001^{*}$} \\
\hline & Non-fluoridated & 5 & $2 \%$ & 0 & $0 \%$ & 3 & $1 \%$ & \\
\hline & Do not know & 101 & $40 \%$ & 30 & $40 \%$ & 126 & $62 \%$ & \\
\hline \multirow{2}{*}{$\begin{array}{l}\text { 6. Do you use } \\
\text { any mouthwash } \\
\text { regularly? }\end{array}$} & Yes & 121 & $49 \%$ & 29 & $39 \%$ & 87 & $42 \%$ & \multirow[t]{2}{*}{$0.193^{* *}$} \\
\hline & No & 127 & $51 \%$ & 46 & $61 \%$ & 119 & $58 \%$ & \\
\hline \multirow{4}{*}{$\begin{array}{l}\text { 7. Do you have } \\
\text { any of the } \\
\text { following oral } \\
\text { habits? }\end{array}$} & Bruxism & 42 & $16.8 \%$ & 9 & $12.0 \%$ & 18 & $8.7 \%$ & \multirow[t]{4}{*}{$0.004^{* *}$} \\
\hline & Clenching & 21 & $8.4 \%$ & 15 & $20.0 \%$ & 42 & $20.4 \%$ & \\
\hline & Biting on a hard object & 103 & $41.2 \%$ & 29 & $38.7 \%$ & 86 & $41.7 \%$ & \\
\hline & Other & 84 & $33.6 \%$ & 22 & $29.3 \%$ & 60 & $29.1 \%$ & \\
\hline
\end{tabular}

Note. All variables are summarized as percentage.

The test of significance was carried out at 0.05 level.

*Monto Carlo test was used.

${ }^{* *}$ Chi- Square test was used.

Significant results are in bold. 


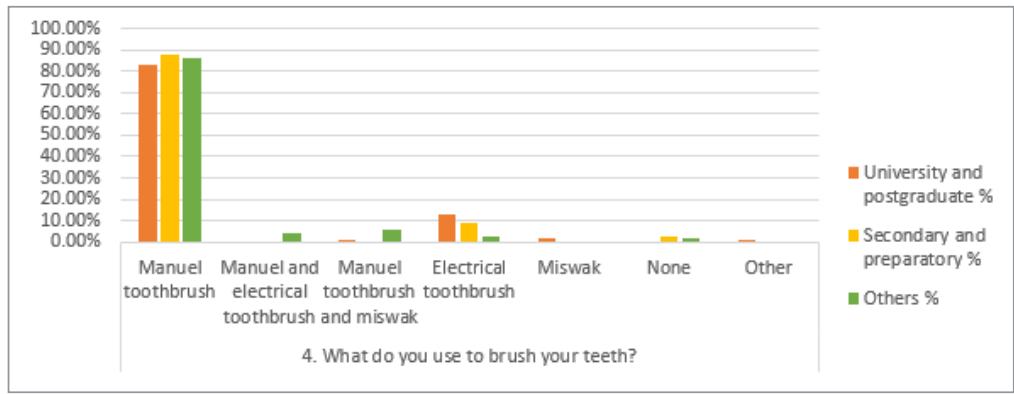

Fig. 9 Toothbrushing way according to mothers' education.

\begin{tabular}{|c|c|c|c|c|c|c|c|c|}
\hline & & \multicolumn{2}{|c|}{$\begin{array}{l}\text { University and } \\
\text { postgraduate }\end{array}$} & \multicolumn{2}{|c|}{$\begin{array}{l}\text { Secondary and } \\
\text { preparatory }\end{array}$} & \multicolumn{2}{|c|}{ Others } & \multirow{2}{*}{ P-value } \\
\hline & & N & $\%$ & $\mathbf{N}$ & $\%$ & $\mathbf{N}$ & $\%$ & \\
\hline \multirow{2}{*}{$\begin{array}{l}\text { 1. Have you ever been } \\
\text { to a dentist? }\end{array}$} & Yes & 246 & $98 \%$ & 72 & $96 \%$ & 198 & $96 \%$ & \multirow[t]{2}{*}{$0.275^{* *}$} \\
\hline & No & 4 & $2 \%$ & 3 & $4 \%$ & 8 & $4 \%$ & \\
\hline \multirow{5}{*}{$\begin{array}{l}\text { 2. If yes, how often do } \\
\text { you visit a dentist? }\end{array}$} & Every 6 months & 45 & $18 \%$ & 19 & $25 \%$ & 40 & $19 \%$ & \multirow[t]{5}{*}{$0.001^{*}$} \\
\hline & Every year & 7 & $3 \%$ & 7 & $9 \%$ & 7 & $3 \%$ & \\
\hline & Irregularly & 57 & $23 \%$ & 5 & $7 \%$ & 38 & $18 \%$ & \\
\hline & Only when in pain & 107 & $43 \%$ & 40 & $53 \%$ & 81 & $39 \%$ & \\
\hline & Other & 34 & $14 \%$ & 4 & $5 \%$ & 37 & $18 \%$ & \\
\hline \multirow{6}{*}{$\begin{array}{l}\text { 3. When was the last } \\
\text { visit? }\end{array}$} & Previous $1-3$ months & 116 & $46 \%$ & 34 & $45 \%$ & 61 & $30 \%$ & \multirow[t]{6}{*}{$0.001 *$} \\
\hline & Previous 4-6 months & 43 & $17 \%$ & 11 & $15 \%$ & 29 & $14 \%$ & \\
\hline & Previous $7-12$ months & 20 & $8 \%$ & 14 & $19 \%$ & 26 & $13 \%$ & \\
\hline & $>1$ year ago & 28 & $11 \%$ & 7 & $9 \%$ & 36 & $17 \%$ & \\
\hline & $>2$ years ago & 24 & $10 \%$ & 6 & $8 \%$ & 24 & $12 \%$ & \\
\hline & $>5$ years ago & 19 & $8 \%$ & 3 & $4 \%$ & 30 & $15 \%$ & \\
\hline \multirow[t]{6}{*}{$\begin{array}{l}\text { 4. What was the } \\
\text { purpose of the visit? }\end{array}$} & $\begin{array}{l}\text { Regular check-up (dental } \\
\text { examination) }\end{array}$ & 57 & $22.8 \%$ & 19 & $25.3 \%$ & 41 & $19.9 \%$ & \multirow[t]{6}{*}{$0.634^{* *}$} \\
\hline & Cleaning the teeth & 36 & $14.4 \%$ & 10 & $13.3 \%$ & 29 & $14.1 \%$ & \\
\hline & Filling & 48 & $19.2 \%$ & 20 & $26.7 \%$ & 46 & $22.3 \%$ & \\
\hline & Extraction & 23 & $9.2 \%$ & 3 & $4.0 \%$ & 25 & $12.1 \%$ & \\
\hline & Orthodontic treatment & 56 & $22.4 \%$ & 13 & $17.3 \%$ & 45 & $21.8 \%$ & \\
\hline & Other & 30 & $12.0 \%$ & 10 & $13.3 \%$ & 20 & $9.7 \%$ & \\
\hline
\end{tabular}

Note. All variables are summarized as percentage.

The test of significance was carried out at 0.05 level.

* Monto Carlo test was used.

**Chi- Square test was used.

Significant results are in bold. 
detailed interviews to overcome internal validity errors that might occur in an electronics-based survey.

\section{Conflict of Interest}

None

\section{References}

1. Leader G, Petersen PE. Chief, Oral Health programme, World Health Organization, Department for Chronic Disease and Health Promotion. Commun Dent Health. 2005;22.

2. Conway DI, McMahon AD, Robertson D, Macpherson LMD. Epidemiology of dental diseases. In: Handbook of Epidemiology: Second Edition. New York: Springer; 2014:2321-53. doi:10.1007/978-0-387-09834-0_51.

3. Halawany HS. A review on miswak (Salvadora persica) and its effect on various aspects of oral health. Saudi Dental J 2012;24(2):63-69. Elsevier. doi:10.1016/j.sdentj.2011.12.004.

4. Levin L, Shenkman A. The relationship between dental caries status and oral health attitudes and behavior in young Israeli adults. J Dent Educ. 2004;68(11):1185-91. doi:10.1002/j.0022-0337.2004.68.11.tb03864.x.

5. Noar SM. A health educator's guide to theories of health behavior. Int $Q$ Commun Health Educ. 2005-2006;24:75-92. doi:10.2190/DALP-3F95GCT3-M922.

6. Christensen P. The health-promoting family: A conceptual framework for future research. Soc Sci Med. 2004;59(2):377-87. doi:10.1016/j. socscimed.2003.10.021

7. Nourijelyani K, Yekaninejad MS, Eshraghian MR, Mohammad K, Foroushan AR, Pakpour AH. The influence of mothers' lifestyle and health behavior on their children: An exploration for Oral Health. Iran Red Crescent Med J. 2014;16(2). Retrieved 12 5:2020. https://ncbi.n/m.nih.gov/pmc/articles/ pmc3965884.

8. Hallas D, Fernandez JB, Lim LJ, Catapano P, Dickson SK, Blouin KR, et al. OHEP: An oral health education program for mothers of newborns. J Pediatr Health Care. 2015;29(2):181-90. Retrieved 12 5, 2020. https://sciencedirect. com/science/article/abs/pii/s0891524514003563.

9. Stillman-Lowe C. Oral Health — Educating mothers with young children. Br Dent J. 2000;188(4):199. Retrieved 12 6. https://nature.com/ articles/4800429
10. Singh A, Purohit B, Sequeira PS, Acharya S. Oral Health status of 5-year-old Aborigine children compared with similar aged marginalised group in south western India. Int Dent J. 2011;61(3):157-62. Retrieved 12 6, 2020. https:// onlinelibrary.wiley.com/doi/10.1111/j.1875-595x.2011.00033.x.

11. Kumar H, Behura SS, Ramachandra S, Nishat R, Dash KC, Mohiddin G. Oral health knowledge, attitude, and practices among dental and medical students in Eastern India - A comparative study. J Int Soc Prev Commun Dent. 2017;7(1):58-63. doi:10.4103/jispcd.JISPCD_30_17.

12. Jaber MF, Khan A, Elmosaad Y, Mustafa MM, Suliman N, Jamaan A. Oral health knowledge, attitude and practices among male Qassim University students. Int J Commun Med Public Health. 2017;4(8):2729. doi:10.18203/2394-6040.ijcmph20173316.

13. McDonald B. Marital Status as a Predictor of Dental Service Utilization. Retrieved 12 6. http://ncurproceedings.org/ojs/index.php/ncur2013/article/ download/419/308, 2020; 2013.

\section{Further reading}

14. Ghofranipour JI, F, Murtomaa H, Murtomaa HInfluence of mothers' oral health knowledge and attitudes on their children's dental health. Eur Arch Paediatr Dent. 2008;9(2):79-83. Retrieved 12 6. https://link.springer.com/ article/10.1007/bf03262614

15. Sayegh A, Dini EL, Holt RD, Bedi R. Caries prevalence and patterns and their relationship to social class, infant feeding and oral hygiene in 4-5-year-old children in Amman, Jordan. Commun Dent Health. 2002;19(3):144-51. Retrieved 12 6, 2020. https://ncbi.nlm.nih.gov/pubmed/12269460.

16. Shah AH, ElHaddad SA. Oral hygiene behavior, smoking, and perceived oral health problems among university students. J Int Soc Prev Commun Dent. 2015;5(4):327-33. 